\title{
SPINAL INFARCT RELATED TO CEREBRAL AMYLOID ANGIOPATHY
}

Authors: $\underline{\text { O. López Agudelo }}^{1}$, L. Redondo Robles ${ }^{1}$, A.D. Murillo Hernández ${ }^{1}$, S. Martínez Peralta ${ }^{1}$, M.D. Calabria Gallego ${ }^{1}$, J. Domínguez Diez¹, M. Álvarez Álvarez¹, A. Sierra Gómez¹, L. López Mesonero', I. Gómez Estévez¹.

${ }^{1}$ Neurology Department, University Hospital of Salamanca, Salamanca, Spain.

\section{BACKGROUND}

Cerebral amyloid angiopathy (CAA) is a cortical and leptomeningeal vessels arteriopathy. CAA spectrum includes intracranial, intraventricular, subarachnoid or subdural hemorrhages. The spinal hemorrhage is a rare manifestation only described in some case reports.

\section{METHOD}

85-year-old man with a personal history of moderately differentiated prostate carcinoma with bone metastasis in T9 treated with hormonotherapy. He was admitted to the hospital due to lumbar pain, paresis of the lower left limb and loss of sphincter control. The next day he was paraplegic and the lumbar pain disappeared. There was no history of fever nor infectious symptoms.

The neurological examination revealed a flaccid paraplegia with muscular stretch reflexes abolished in the lower limbs, the cutaneous-abdominal reflexes were present, the cutaneous-plantar reflexes were indifferent, the sensitivity was preserved and there was loss of sphincter control.

\section{RESULTS}

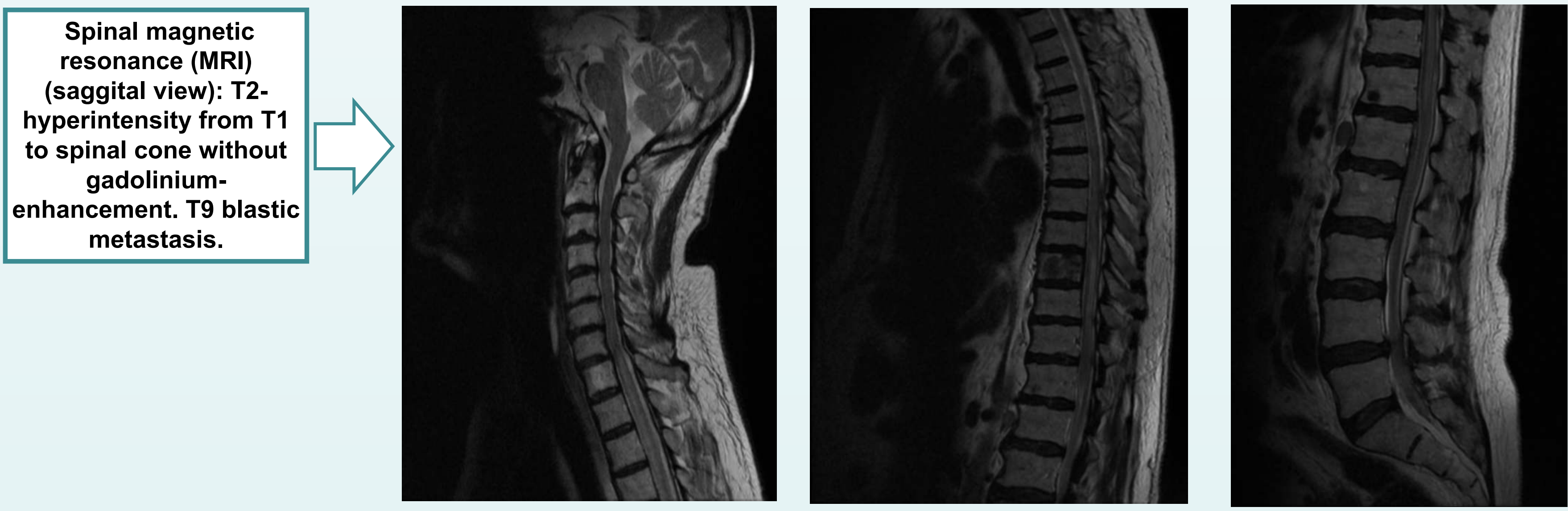

Cranial computed tomography (axial view): parietal bilateral subarachnoid hemorrhage with intraventricular component
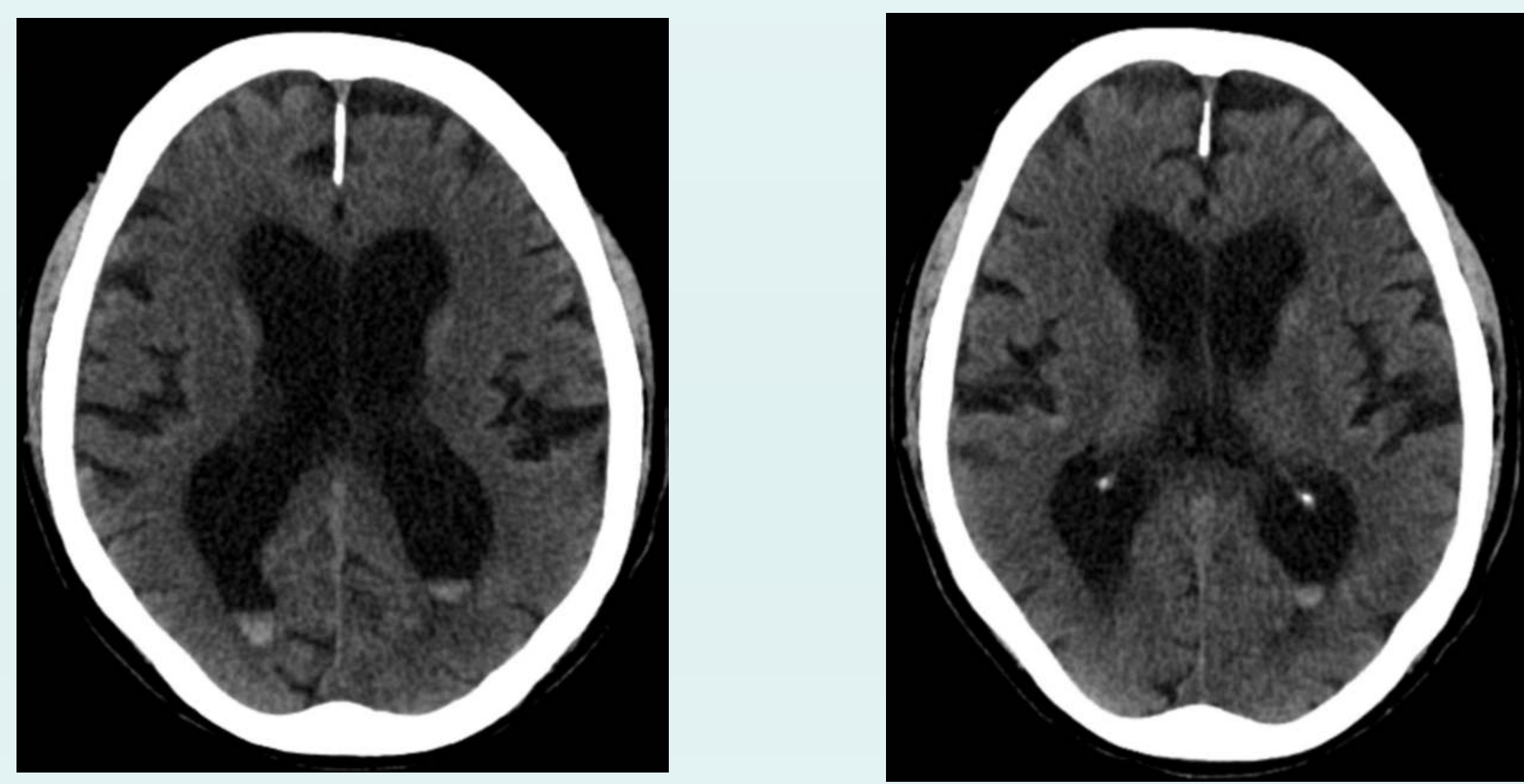

Cranial angiography MRI as well as medullar and vertebral arteriography ruled out vascular malformations. The lumbar puncture revealed a hemorrhagic cerebrospinal fluid

\section{CONCLUSIONS}

The spinal infarct in CAA has not been described before. CAA has been documented in a minority of meningeal spinal vessels and has not been observed in the parenchymal spinal cord vessels. We believe that the spinal infarct was due to the vasospasm of the anterior spinal artery induced by the blood in the subarachnoid space in the 